\title{
Strafrechtsprofessoren für eine Reform des Betäubungsmittelstrafrechts: ein Projekt
}

\section{A. Drogenpolitik im Wege der Aktionsforschung}

$\mathrm{Zu}$ berichten ist über ein von mir im Mai 2010 begonnenes kriminalpolitisches Forschungsprojekt. Das Projekt folgt der Methode der Aktionsforschung (Lewin 1948) und bezieht sich auf die soziologische Akteur-Netzwerk-Theorie (Latour 2007). Mitträger des Projekts ist der „Schildower Kreis“, ein auf Drogenpolitik bezogenes interdisziplinäres Netzwerk von Experten aus Wissenschaft und Praxis.

Kurz zur Methode der Aktionsforschung, auch Handlungsforschung: diese Begriffe sind Übersetzungen des von Kurt Lewin geprägten Begriffs, action research'. Selbst ein Pionier der Sozialpsychologie, kritisierte Lewin die rein experimentelle Sozialpsychologie. Nach seiner Auffassung sollten Grundannahmen und Hypothesen der Wissenschaft an der sozialen Realität orientiert und praxisnah sein. Als Gegenentwurf zu einer „reinen“ und potentiell verantwortungslosen Wissenschaft sollten Erkenntnisse zu Veränderungen und Problemlösungen führen. Auch "Laien" sind beteiligt und erweitern, korrigieren dadurch kontinuierlich die Perspektive der professionell Forschenden. Ziel der Forschung ist es, mittels intervenierender Aktion ein relevantes gesellschaftliches Problem anzugehen. Die Aktion und ihre methodische Verarbeitung generieren neue Erkenntnisse hinsichtlich der Wirksamkeit der Intervention selber und der zugrunde liegenden gesellschaftlichen Strukturen.

Nun zur Vorgehensweise: Mit folgendem, an sämtliche mir durch die Strafrechtlehrervereinigung zugängliche Email-Adressen versandten Brief appellierten wir, die beigefügte Resolution zu unterzeichnen:

Initiative „Einrichtung einer Enquête-Kommission des Bundestages zum Thema ,Erwünschte und unbeabsichtigte Folgen des geltenden Drogenstrafrechts' " (Sprecher: Lorenz Böllinger)

Resolution deutscher Strafrechtsprofessorinnen und -professoren an die Abgeordneten des Deutschen Bundestages

A. Notwendigkeit der Überprüfung der Wirksamkeit des Betäubungsmittelgesetzes

Die Unterzeichnenden wollen den Gesetzgeber auf die unbeabsichtigten schädlichen

Nebenwirkungen und Folgen der Kriminalisierung bestimmter Drogen aufmerksam machen. Sie wollen das Parlament anregen, bezüglich dieser Thematik seinem verfassungsrechtlichen Auftrag im Allgemeinen und den wissenschaftlich begründeten Prin- 
zipien von Strafgesetzgebung und Kriminalpolitik im Besonderen durch die Einrichtung einer Enquête-Kommission Rechnung zu tragen. Sowobl aus strafrechtswissenschaftlicher Sicht als auch aufgrund empirischer Forschungsergebnisse besteht die dringende Notwendigkeit, die Geeignetheit, Erforderlichkeit und normative Angemessenheit des Betäubungsmittelstrafrechts zu überprüfen und gegebenenfalls Vorschläge $z u$ Gesetzesänderungen aus solcher Evaluation abzuleiten.

Eine solche Initiative mag vielen von Ibnen unangebracht und aussichtslos erscheinen, wo doch das Bundesverfassungsgericht in seiner Cannabis-Entscheidung 1994 die Verfassungsmäßigkeit des geltenden Betäubungsmittelstrafrechts grundsätzlich bestätigt hat. Gleichwobl gibt es aus unserer Sicht nabezu 20 Jahre danach Anlass diese Thematik neuerlich auf die rechtspolitische Agenda zu setzen. Zum einen zeigt sich weltweit die Erfolglosigkeit strafrechtlicher Bekämpfung von Drogennachfrage und -angebot. Zum anderen sind derzeit Auswüchse der Kriminalisierung zu beobachten, welche erst recht parlamentarisches Nachdenken erfordern. Nur zwei Beispiele: Die Finanzierung des Taliban-Terrorismus in und aus Afghanistan erfolgt allen Erkenntnissen zufolge weitgehend über den Schwarzmarkt mit Heroin und Haschisch. Und: Tausende von Toten in dem aktuellen "Krieg der Drogenkartelle" in Mexiko sind weitgehend den Kartellkämpfen um exorbitante Profite auf dem Schwarzmarkt zuzurechnen. Der Schwarzmarkt generiert eine extreme und globalisierte Schattenwirtschaft mit weiterer Folgekriminalität und destabilisierenden Auswirkungen auf globale Finanzmärkte ebenso wie nationale Volkswirtschaften. Angesichts effektiver informeller Geldtransfersysteme (z.B. Hawala-System) kann Geldwäschekontrolle nicht funktionieren. Demgegenüber zeigen alle wissenschaftlichen Erkenntnisse, dass die Gefährdungen durch bislang illegale Drogen ebenso wie solche durch Medikamente und Alkohol besser durch gesundheitsrechtliche Regulierung mit akzessorischer ordnungs- oder strafrechtlicher Sanktionierung sowie mit adäquaten Jugendschutz und - bilfemaßnahmen zu bewältigen wären.

Ein weiterer Anlass für unsere Initiative: diverse Quasi-Feldexperimente mit der liberalisierten Zugänglichkeit oder Vergabe von bislang illegalen Drogen (z.B. Niederlande, Schweiz, Spanien, Portugal) ergaben, dass die befürchtete Ausweitung des Drogenkonsums ausbleibt. Außerdem hat sich das drogenpolitische Klima in den bislang im repressiven Drogenregime federfübrenden U.S.A. stark zu verändern begonnen. Beispielhaft seien genannt: am 10.12.2009 die Einrichtung eines Ausschusses des Repräsentantenhauses zur Untersuchung des Scheiterns der Drogenpolitik, diverse auf YOUTUBE anzusehende Reden von U.S.-Professoren und -Polizeifunktionären (siebe Links www.schildower-kreis.de), die am 2.11. stattfindende Volksbefragung zur Freigabe von Cannabis ("Proposition 19") in Kalifornien, die zunehmende Legalisierung von Cannabis als Medizin (bislang in 16 U.S.-Bundesstaaten). Die Obama-Regierung hat stillschweigend den Paradigmen-Wechsel vom "Krieg gegen die Drogen" zu gesundheitspolitischen Strategien vollzogen.

Die Notwendigkeit der Einrichtung einer Enquête-Kommission des Bundestages ergibt sich daraus, dass der Gesetzgeber gemäß dem allgemeinen Verhältnismäßigkeitsprinzip der Verfassung hinsichtlich geltender Gesetze eine Überprüfungspflicht hat 
und auf deutliche Veränderungen in der sozialen Wirklichkeit und in der Wissenschaft reagieren muss.

\section{B. Thesen zur Begründung}

Die strafrechtliche Drogenprobibition ist gescheitert, sozialschädlich und unökonomisch.

1. Mit der Drogenprobibition gibt der Staat seine Kontrolle über Verfügbarkeit und Reinbeit von Drogen auf.

Nicht die Wirkung der Drogen ist das Problem, sondern die repressive Drogenpolitik schafft Probleme. Die überwiegende Zabl der Drogenkonsumenten lebt ein normales Leben. Selbst abbängige Konsumenten bleiben oftmals sozial integriert. Menschen mit problematischem Drogenkonsum brauchen Hilfe. Die Strafverfolgung hat für sie und alle anderen nur negative Folgen.

2. Der Zweck der Probibition wird systematisch verfeblt.

Probibition soll den schädlichen Konsum bestimmter Drogen verbindern. Tatsächlich kann sie dieses Ziel nicht erreichen. Das zeigen alle wissenschaftlich relevanten Untersuchungen. Sogar die Evaluation des 10-Jahres-Programms der UNO zur Drogenbekämpfung kommt im Jahr $2008 \mathrm{zu}$ diesem Schluss.

Probibition schreckt zwar einige Menschen ab, verbindert aber Aufklärung und vergrößert gleichzeitig dramatisch die gesundheitlichen und sozialen Schäden für diejenigen, die nicht abstinent leben wollen. Selbst in totalitären Regimen und Strafanstalten kann Drogenkonsum nicht verbindert werden.

3. Die Probibition ist schädlich für die Gesellschaft.

Sie fördert die organisierte Kriminalität und den Schwarzmarkt.

Sie schränkt Bürgerrechte ein und korrumpiert den Rechtsstaat. Durch massive Machtanballung bei Kartellen und Mafia nimmt die Gefabr eines Scheiterns der Zivilgesellschaft zu. Stimuliert durch gigantische Profite aus dem Drogenschwarzmarkt entsteben veritable Kriege zwischen Drogenkartellen und in Reaktion darauf sowobl eine Quasi-Militarisierung der Polizei als auch quasi-polizeiliche Funktionen des Militärs. Auch dadurch erodieren staatliche Grundstrukturen.

Sie hat desaströse Auswirkungen auf Anbau-und Transitländer.

Sie bebindert eine angemessene medizinische Versorgung.

4. Die Probibition ist unverbältnismäßig kostspielig

Die Bürger werden Opfer der Beschaffungskriminalität.

Jedes Jahr werden Milliardenbeträge für die Strafverfolgung aufgewendet, welche sinnvoller für Prävention und Gesundheitsfürsorge eingesetzt werden könnten.

Der Staat verzichtet auf Steuereinnahmen, die er bei einem legalen Angebot hätte.

\section{Die Probibition ist schädlich für die Konsumenten}

Konsumenten werden diskriminiert, strafrechtlich verfolgt und in kriminelle Karrieren getrieben. Weil es sich um "opferlose “ Kontrolldelikte handelt, welche lediglich proaktiv - und damit Unterschichtangehörige und Migranten benachteiligend-verfolgt werden. 
Es gibt keinen Verbraucher- und Jugendschutz. Riskante Konsumformen werden gefördert und die Konsumenten werden gefährlichen Krankheiten ausgesetzt (z.B. AIDS, Hepatitis C).

Normales jugendliches Experimentierverhalten wird kriminalisiert und das Erlernen von Drogenmündigkeit erschwert. Junge Menschen werden dauerhaft stigmatisiert und ibre Lebenschancen werden gemindert.

C. Fazit

Der Staat darf die Bürger durch die Drogenpolitik nicht schädigen. Es ist deshalb notwendig, Schaden und Nutzen der Drogenpolitik unvoreingenommen wissenschaftlich zu überprüfen.

Als Kriminalwissenschaftler füblen wir uns in besonderem Maße verantwortlich für die Einhaltung strafrechtstheoretischer Prinzipien und für die Zurückbaltung des Staates in der Anwendung der ultima ratio gesellschaftlicher Stenerung.

Deshalb appellieren wir an die Abgeordneten des Deutschen Bundestages, nicht nur dem Fraktionszwang zu folgen, sondern auch ibrer individuellen Verantwortung.

Für den Initiativkreis: Prof. Dr. L. Böllinger (Sprecher)

\section{B. Zielsetzung des Projekts}

Beabsichtigt ist, durch solchen strafrechtswissenschaftlichen ,impact' mindestens ein Viertel der Abgeordneten zur Beantragung einer Enquète-Kommission zu veranlassen. Im Sinne der verfassungsrechtlich vorgegebenen Überprüfungspflicht des Gesetzgebers soll diese eine wissenschaftlich und interdisziplinär begründete Evaluation des Drogenstrafrechts vornehmen. Fernziel ist die Veränderung des Drogenstrafrechts auf der Grundlage empirischer Forschung und informierter gesellschaftlicher bzw. parlamentarischer Verständigung. Solche hat bisher das BtMG betreffend nie stattgefunden.

Der mittels der Enquête-Kommission an den Gesetzgeber adressierte Appell ist in mehrfacher Hinsicht verfassungsrechtlich begründet. Die umfassendste Begründung findet er im zentralen Prinzip des Grundgesetzes, dem von allen drei Teilgewalten zugrunde zu legenden Verbältnismäßigkeitsprinzip. Daraus ergibt sich allgemein und formal, dass Gesetze, welche die Grundfreiheiten der Bürger einschränken, inhaltlich und wissenschaftlich begründet sein müssen und dass sie im Verlauf ihrer Anwendung hinsichtlich ihrer Wirksamkeit überprüft werden müssen. Dogmatisch operationalisiert ist das Verhältnismäßigkeitsprinzip nach allseits akzeptierter Verfassungslehre in den drei Unterprinzipien „Erforderlichkeit“, „Geeignetheit“ und „Proportionalität“. In diesem Rahmen ist die inhaltliche Überprüfung des BtMG vorzunehmen.

Das BVerfG hat zwar in seiner Cannabis-Entscheidung von 1994 und in mehreren darauf basierenden Nicht-Annahme-Beschlüssen die strafrechtlichen Vorschriften des BtMG für verfassungsgemäß befunden. Es legte jedoch in enger exemplarischer Auswahl die damals aktuelle, lückenhafte Daten- und Erkenntnislage zugrunde. Es beachtete nicht, dass der BtM-Gesetzgeber 1971 auf Druck der USA ohne eigene wissenschaftliche Begründung unbesehen die Vorgaben der UNO-Single Convention von 1961 in das 
BtMG umgesetzt hatte. In den seither verstrichenen fast $20 \mathrm{Jahren}$ hat sich zum einen die soziale Realität in hohem Maße verändert, zum anderen die entsprechende wissenschaftliche Daten-, Methoden und Erkenntnislage massiv erweitert. Neubewertung und NeuInitiierung entsprechender Forschung sind also erforderlich, um dem BtMG erstmals eine dem Verfassungs- und Gesetzgebungsrecht genügende Grundlage zu verleihen. Die fünf Thesen zur Begründung der Resolution könnten auch Grundlage eines systematischen Prozederes der eventuellen Enquête-Kommission des Bundestages werden.

Die Strafrechtsbewehrung des Umgangs mit als illegal definierten Drogen ist nicht mehr zeitgemäß. Sie verstößt gegen das Verhältnismäßigkeitsprinzip des Grundgesetzes, insbesondere gegen das Unterprinzip der Geeignetheit (Nestler 2010). Sie ist auch nicht erforderlich, denn für den lediglich von einer Teilpopulation der Drogenkonsumenten praktizierten gefährlichen oder selbstschädigenden Umgang mit diesen Substanzen gibt es geeignete, nicht-strafrechtliche, gesundheitssystemische Abhilfen (Haffke 1995).

Die Strafbarkeit des Umgangs mit BtM widerspricht im Übrigen auch einer zentralen strafrechtstheoretischen Maxime: Strafe als äußerstes Mittel der Lösung von sozialen und zwischenmenschlichen Problemen darf nur für Situationen erheblicher Fremdschädigung angedroht werden, nicht jedoch für von informiertem Einverständnis getragene Selbstschädigung. Die Logik und Dogmatik der Gefährdungsdelikte wird missbraucht, wenn der Gesetzgeber behauptet, die „Volksgesundheit“ und das „Soziale Zusammenleben“ durch empfindlich Strafdrohungen schützen zu müssen: Jeder mündige Konsument konsumiert im Rahmen eigener Willensfreiheit - auch wenn er sich dabei selbst schädigt (Hassemer 1993; Nestler 1997). Solche Schädigung betrifft aber nur den geringen Teil der Drogenkonsumenten, welche den Gebrauch nicht steuern können. Im Übrigen sind die Schäden ja gerade durch die Prohibition und den daraus resultierenden Schwarzmarkt und Drogenkrieg verursacht.

Nur eine rationale, gesundheitsrechtliche und soziale Drogenpolitik durch substanzspezifische und wissenschaftlich begründete Legalisierung sowie Prävention und Behandlung kann das Problem in relevantem Ausmaß mindern (Gerlach/Stöver 2012).

\section{Verlauf des Aktionsforschungsprojekts}

Es meldeten sich im ersten Anlauf ca. 40 Professorinnen und Professoren. Seither haben wir, insbesondere jeweils nach Erhalt der jährlich aktualisierten Mitgliederliste der von Prof. Schünemann dankenswerter Weise vorbildlich betreuten Strafrechtslehrervereinigung, vier weitere „Kampagnen“ gestartet, zuletzt Anfang 2013. Viele Kolleginnen und Kollegen hatten die Email zunächst übersehen, nicht gelesen oder als Spam betrachtet. Peu à peu sind letztlich 122 UnterzeichnerInnen zusammengekommen, weit über ein Drittel der ca. 300 deutschen Strafrechtsprofessorinnen und -professoren. Auch ein ehemaliger Richter des BVerfG, die Neue Richtervereinigung als Ganze sowie die Strafverteidigervereinigung Hessen haben sich angeschlossen.

Acht Strafrechtsprofessoren lehnten die Unterschrift mit der Begründung ab, sie könnten sich mit den Aussagen der Resolution nicht identifizieren. Sie akzeptierten nicht, 
dass eine derartige Festlegung mit dem Appell eine Enquête-Kommission zu beantragen nicht verbunden war: das Ergebnis der Beratungen einer solchen Kommission bliebe jedenfalls offen. Die übrigen angeschriebenen Kolleginnen und Kollegen reagierten nicht.

Entsprechend der Planung wurden nach der Bundestagswahl 2013 die einzelnen Bundestagsfraktionen als Ganze, die Drogenbeauftragten der Parteien und - soweit bekannt - einzelne BT-Abgeordnete per Email und mit dem Resolutionstext im Anhang angeschrieben. Sie wurden gebeten, einen entsprechenden Antrag auf Einrichtung einer „Enquête-Kommission Evaluation der Drogenpolitik“ aus der Mitte des Parlaments zu unterstützen. Gemäß \56 BT-Geschäftsordnung muss eine solche Kommission eingesetzt werden, wenn 25\% der Mitglieder des BT dies beantragen. Mittlerweile hat die BTMehrheit zugesagt, der Minderheit dieses Recht zu garantieren, obwohl die Opposition lediglich über ca. $20 \%$ der Stimmen verfügt.

Flankiert wurde diese Aktion durch frühzeitige Kontakte zu kritischen Medien und zur Fachpresse. Mittlerweile hat es eine Vielzahl von Zeitungs-, TV- und Rundfunkinterviews durch einige der Unterzeichner gegeben.

\section{Diskussion des Projekts und seiner Aussicht}

Wie sind nun die Aussichten dieses laufenden Projekts der Aktionsforschung?

Die Sache kann zunächst schon daran scheitern, dass 20\% der Abgeordneten im neuen Bundestag nicht zu gewinnen sein könnten. Im Februar 2014 fanden Gespräche im BT statt, in denen die Fraktionen der GRÜNEN und der LINKEN zusagten, ihre Abgeordneten für diesen Antrag zu gewinnen. Seitens CDU und SPD kam keine Reaktion. Wir bemühen uns weiter um direkte Abgeordnetenkontakte. Die Sache könnte durch aktuelle politische Entwicklungen gefährdet sein: zum einen bindet womöglich der NSAUntersuchungsausschuss die Kräfte; zum anderen hat sich über den Ukraine-Konflikt eine Kluft zwischen der LINKEN und den GRÜNEN entwickelt. Schließlich ist das Ergebnis einer solchen Kommission völlig offen: Die entsprechend dem BT-Mehrheitsverhältnis zusammengesetzte Kommission könnte den Auftrag verändern, und verwässern. Insbesondere über die Benennung der anzuhörenden Experten könnte auf das Ergebnis Einfluss genommen werden. Schließlich sind die wissenschaftliche Erkenntnis regelmäßig beiseite schiebenden Mechanismen des politischen Aushandelungsprozesses im Parlament einzukalkulieren. Die Verhandlungen könnten auf eine Zementierung des status quo hinauslaufen.

Gleichwohl könnte positiv wirken, dass Enquête-Kommissionen eher geeignet erscheinen und in der Vergangenheit installiert wurden für Themen, wo die inhaltlichen Differenzen quer durch alle Parteien gehen. Zu beachten sind auch die Mechanismen der Beeinflussung von Gesetzgebung durch Medien und Politik. Hierdurch kann die deutliche Veränderung der globalen Diskussion und Rechtslage gegen den Widerstand der aktuellen, das Drogenproblem totschweigenden Großen Koalition in die Debatte gebracht werden. Schon die Anregung eines Gesetzesexperiments zur Erprobung eines 
alternativen Drogenkontrollsystems mit entsprechender Evaluation wäre der optimale Erfolg dieses Experiments.

In jedem Falle wird dieses Experiment in action research aus forscherischer Sicht interessant sein. Insbesondere auf dem Hintergrund der Akteur-Netzwerk-Theorie (Latour 2007) verspricht die Auswertung einen Gewinn. Kerngedanke dieser Theorie ist ja, dass die Welt netzwerkartig, assoziativ und interaktionell verfasst ist, wobei von einem Zusammenschluss unterschiedlicher Elemente zu mehr oder weniger kohärenten Akteuren personeller, institutioneller und auch natürlicher Art ausgegangen wird. Diese vernetzen sich und interagieren zum einen auf je horizontaler, aktueller gesellschaftlicher Ebene, zum anderen gleichsam vertikal, im historischen Verlauf der materiellen Determination von Gegenwart durch Vergangenes. Diese Theorie kann - ganz im Sinne des Konstruktivismus - insbesondere auch die immaterielle Interaktivität und Intersubjektivität von Subjektivität, von Bedeutungszuschreibungen und deren Wandel, von unbewussten und gruppendynamischen Motiven der Realitätswahrnehmung und ihrer Verzerrungen, der nachträglichen, von Interessen und Machtverhältnissen geleiteten Umdeutung von gesellschaftlichen Phänomenen - also eine Verbindung der Makro- und Mikro-Ebene erfassen.

Im Vorgriff auf den weiteren, noch unklaren Verlauf des Projekts einige Hypothesen: Aktuelle Drogenpolitik stellt ein in 45 Jahren gewachsenes, verfestigtes Netzwerk aus Traditionen, Interessen, Machtverhältnissen dar, welches durch rationale Formen der Politikberatung nicht zu beeinflussen ist. Die Repräsentanten und Akteure sind jeweils verstrickt in Konformitätsdruck und wechselseitige Abhängigkeiten. Auch unbewusste Ängste als gegen zentrale gesellschaftliche Arbeitstugenden, als ,Jugendverführer' und von eigenen Interessen geleitet denunziert zu werden, tragen zur öffentlichen Leugnung von privat erkannter Widersprüchlichkeit und Sinnlosigkeit aktueller Drogenpolitik bei.

\section{Literatur}

Gerlach E Stöver (Hrsg.) (2012) Entkriminalisierung von Drogenkonsumenten - Legalisierung von Drogen

Haffke (1995) Drogenstrafrecht, ZStW 1995,761 ff.

Hassemer Entkriminalisierung im Betäubungsmittelstrafrecht, KritV 1993, $198 \mathrm{ff}$.

Latour (2007) Eine nene Soziologie für eine neue Gesellschaft. Einführung in die AkteurNetzwerk-Theorie. Aus dem Englischen von Gustav Roßler (Originalausgabe: Reassembling The Social. Oxford University Press, 2005).

Lewin (1948) Aktionsforschung und Minderheitenprobleme, in K. Lewin (Ed.), Die Lösung sozialer Konflikte (pp. 278-298)

Nestler (1997) Grundlagen und Kritik des Betäubungsmittelstrafrechts, in: Kreuzer (Hrsg.), Handbuch des Betäubungsmittelstrafrechts, $\ 11$ 
Nestler (2010) Geeignetheit und das strafrechtliche Verbot des Umgangs mit Cannabis - eine Projektskizze, in: Herzog / Neumann (Hg.) Fschrf. Winfried Hassemer, S. $971-984$

Tondorf (2013) Die Entkriminalisierung von DrogenkonsumentInnen, in: Fschr. Michael Walter

Kontakt:

Prof. Dr. jur. Lorenz Böllinger, Dipl.-Psych.

Em. Prof.f. Strafrecht u. Kriminologie

Appr.Psychotherapeut / Psychoanalytiker (DPV/IPA)

Donandtstr. 80

D-28209 Bremen

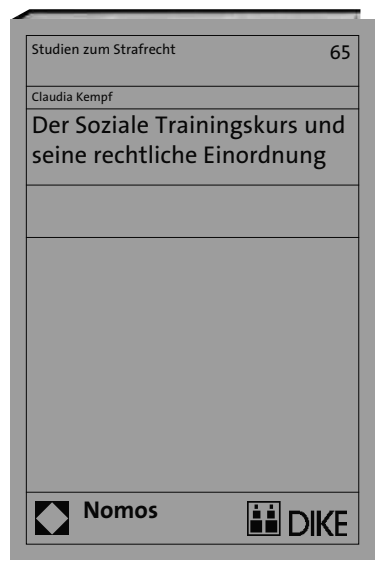

\section{Der Soziale Trainingskurs und seine rechtliche Einordnung}

Von Dr. Claudia Kempf

2014, 291 S., brosch., 79,- -€

ISBN 978-3-8487-1391-2

(Studien zum Strafrecht, Bd. 65)

www.nomos-shop.de/22668

Diese Arbeit will dem Manko abhelfen, dass kaum ein (Jugend-)Richter oder Staatsanwalt genaue Vorstellungen vom Geschehen in Sozialen Trainingskursen für straffällige Jugendliche hat. Dazu hat die Autorin an sechs derartigen Maßnahmen beobachtend teilgenommen und einige markante Vorgänge einer kritischen rechtlichen Würdigung unterzogen.

Bestellen Sie jetzt telefonisch unter 07221/2104-37 Portofreie Buch-Bestellungen unter www.nomos-shop.de Alle Preise inkl. Mehrwertsteuer

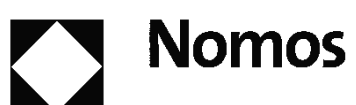

